\title{
On Birkhoff Interpolations with Fractional-order Derivatives
}

\author{
Tinggang Zhao \\ School of Mathematics, Lanzhou City University, Lanzhou, Gansu, China \\ Email: 13669397938@163.com
}

\begin{abstract}
In this paper, we consider some interpolations of Birkhoff-type with fractional-order derivative. The Birkhoff interpolations is related with collocation method for the corresponding initial or boundary value problems of differential equations of fractional-order. The solvability of the interpolation problems is studied. For Gauss-type interpolating points, error of interpolation approximation is deduced.
\end{abstract}

Keywords: Birkhoff interpolation, collocation method, fractional calculus.

\section{Introduction}

Back to 1906, an extension of polynomial interpolation which involves the values of derivatives of the interpolated function was studied by G. D. Birkhoff in [1]. The Birkhoff interpolation (named after G. D. Birkhoff) may be defined as follow. Given a set of distinct interpolating points $\left\{x_{j}\right\}_{j=0}^{N}$ and $K+1$ data $\left\{y_{j}^{m}\right\}_{j=0}^{N}(K \geq N)$, to find a polynomial $p(x)$ of degree $K$ such that

$$
p^{(m)}\left(x_{j}\right)=y_{j}^{m} .
$$

If for each $j$, the orders of derivatives in (1) form a unbroken sequence, $m=0,1, \ldots, m_{j}$, the interpolation problem above refers to Hermite interpolation. But in general, we say the Birkhoff interpolation which means the other case. It turns out that the Birkhoff interpolation problem is difficult and causes many literatures $[2,3,4,5,6,7]$. In contrast to Lagrange interpolation and Hermite interpolation, a Birkhoff interpolation problem does not always have a unique solution. For instance, there is no quadratic polynomial $p$ such that $p(-1)=p(1)=0$ and $p^{\prime}(0)=1$. For solvability of Birkhoff interpolation problems, Pólya condition is suggested in [8] and developed in [9]. To construct the Birkhoff interpolation formulation, an algorithmic approach is presented in [10] for Hermite-Birkhoff interpolation. There are also many results for $(0,2)$ interpolation [11] and others.

In any way, the approximation theory of interpolation is of foundational importance in numerical analysis especially for various scientific computing problems. In [12], authors suggested a well-conditioned collocation method based on a Birkhoff interpolation which achieved efficiency in computation. Newly in [13], the fractional Birkhoff interpolation was suggested which promised a good prospect for solving fractional-order differential equations by well-conditioned collocation method. It is necessary to contemplate the Birkhoff interpolation presented in publications mentioned above.

There are two contributions in this work. (1) The fractional Birkhoff interpolations on common interpolating nodes are studied, especially the solvability of the problems. (2) We give the error estimates of the fractional Birkhoff interpolation at Gauss-Jacobi-points. The approximation results give theoretical confirmation of their applications to the corresponding problem-solving.

This paper is organized as follow. In sect 2 , we present some definitions of fractional calculus. In sect 3 , we present the concerned interpolation problems. In sect 4 , we give solvability of the problems. In sect 5 , we present the interpolation errors. In sect 6 , some conclusive remarks are given.

\section{Definitions of Fractional Calculus}

In this section, we introduce some definitions of fractional-order calculus. There are various fractional-order derivatives (see [14] or others). Riemann-Liouville's and Caputo's are more popular. 
Denote by $I^{\mu}(0<\mu<1)$, the left Riemann-Liouville fractional integral, which is defined as:

$$
\left(I^{\mu} u\right)(x)=\frac{1}{\Gamma(\mu)} \int_{-1}^{x} \frac{u(t)}{(x-t)^{1-\mu}} \mathrm{d} t, \quad x \in[-1,1] .
$$

Denote by $D^{\mu}(m-1<\mu<m)$, the left Riemann-Liouville fractional derivative, which is defined as:

$$
\left(D^{\mu} u\right)(x)=\frac{d^{m}}{d x^{m}} I^{m-\mu} u(x)=\frac{1}{\Gamma(m-\mu)} \frac{d^{m}}{d x^{m}} \int_{-1}^{x} \frac{u(t)}{(x-t)^{\mu-m+1}} \mathrm{~d} t, \quad x \in[-1,1] .
$$

Denote by ${ }^{C} D^{\mu}(m-1<\mu<m)$, the left Caputo fractional derivative, which defined as:

$$
\left({ }^{C} D^{\mu} u\right)(x)=I^{m-\mu} \frac{d^{m}}{d x^{m}} u(x)=\frac{1}{\Gamma(m-\mu)} \int_{-1}^{x} \frac{u^{(m)}(t)}{(x-t)^{\mu-m+1}} \mathrm{~d} t, \quad x \in[-1,1] .
$$

The definitions mentioned above are referred to as left-side. There are also definitions of right-side fractional-order calculus and the results are similar to those in this paper.

\section{Fractional Birkhoff Interplations}

Without loss of generality, we always take the interpolating nodes $\left\{x_{j}\right\}_{j=1}^{N} \subset(-1,1)$. Denote by $\mathcal{P}_{N}$ the space of all algebraic polynomials with degree at most $N$.

\subsection{Caputo Fractional Birkhoff Interpolating Problems}

Consider the following two Caputo fractional Birkhoff interpolating problems [13]:

1. For $\mu \in(0,1)$, the interpolation of any $u \in \mathcal{C}_{1}$, denoted by ${ }^{C} I_{N}^{\mu} u$, is a polynomial such that ${ }^{C} I_{N}^{\mu} u \in \mathcal{P}_{N}$ and

$$
{ }^{C} D^{\mu}\left({ }^{C} I_{N}^{\mu} u\right)\left(x_{j}\right)=\left({ }^{C} D^{\mu} u\right)\left(x_{j}\right), 1 \leq j \leq N ;{ }^{C} I_{N}^{\mu} u(-1)=u(-1) .
$$

where $\mathcal{C}_{1}=\left\{u: u \in C[-1,1]\right.$ and $\left.{ }^{C} D^{\mu} u \in C(-1,1]\right\}$.

2. For $\mu \in(1,2)$, the interpolation of any $u \in \mathcal{C}_{2}$, denoted by ${ }^{C} I_{N}^{\mu} u$, is a polynomial such that ${ }^{C} I_{N}^{\mu} u \in \mathcal{P}_{N}$ and

$$
{ }^{C} D^{\mu}\left({ }^{C} I_{N}^{\mu} u\right)\left(x_{j}\right)=\left({ }^{C} D^{\mu} u\right)\left(x_{j}\right), 1 \leq j \leq N-1 ;{ }^{C} I_{N}^{\mu} u( \pm 1)=u( \pm 1) .
$$

where $\mathcal{C}_{2}=\left\{u: u \in C[-1,1]\right.$ and $\left.{ }^{C} D^{\mu} u \in C(-1,1)\right\}$.

\subsection{Riemann-Liouville Fractional Birkhoff Interpolating Problems}

By replacing Caputo derivative with Riemann-Liouville's, we give two Riemann-Liouville fractional Birkhoff interpolating problems [13]:

1. For $\mu \in(0,1)$, the interpolation of any $u \in \mathcal{D}_{1}$, denoted by $I_{N}^{\mu} u$, is a polynomial such that $I_{N}^{\mu} u \in \mathcal{P}_{N}$ and

$$
D^{\mu}\left(I_{N}^{\mu} u\right)\left(x_{j}\right)=\left(D^{\mu} u\right)\left(x_{j}\right), 1 \leq j \leq N ; I_{N}^{\mu} u(-1)=u(-1) .
$$

where $\mathcal{D}_{1}=\left\{u: u \in C[-1,1]\right.$ and $\left.D^{\mu} u \in C(-1,1]\right\}$.

2. For $\mu \in(1,2)$, the interpolation of any $u \in \mathcal{D}_{2}$, denoted by $I_{N}^{\mu} u$, is a polynomial such that $I_{N}^{\mu} u \in \mathcal{P}_{N}$ and

$$
D^{\mu}\left(I_{N}^{\mu} u\right)\left(x_{j}\right)=\left(D^{\mu} u\right)\left(x_{j}\right), 1 \leq j \leq N-1 ; I_{N}^{\mu} u( \pm 1)=u( \pm 1) .
$$

where $\mathcal{D}_{2}=\left\{u: u \in C[-1,1]\right.$ and $\left.D^{\mu} u \in C(-1,1)\right\}$.

Obviously, it is unnecessary for the interpolating points corresponding to the above mentioned fractional Birkhoff problems to be Jacobi-Gauss-type. We present the solvability of those problems without emphasis. 


\section{Solvability of the Fractional Birkhoff Problems}

For Caputo fractional derivative operator, we have the following formulas [14]: for $\mu>0$ and not an integer,

$$
{ }^{C} D^{\mu}(x+1)^{n}= \begin{cases}0, & \text { if } n<\mu \text { is a nonnegative integer; } \\ \frac{n !}{\Gamma(n-\mu+1)}(x+1)^{n-\mu}, & \text { if } n>\mu \text { is a nonnegative integer. }\end{cases}
$$

Consider the Caputo fractional Birkhoff interpolation of $u \equiv 0$. In the case $\mu \in(0,1)$, if we express the interpolation polynomial as

$$
p(x)=\sum_{k=0}^{N} a_{k}(x+1)^{k}, \quad p(x) \in \mathcal{P}_{N}
$$

then we have by $(9)$

$$
{ }^{C} D^{\mu} p(x)=\sum_{k=0}^{N}{ }^{C} D^{\mu}\left(a_{k}(x+1)^{k}\right)=(x+1)^{1-\mu} \sum_{k=0}^{N-1} a_{k+1} \frac{(k+1) !}{\Gamma(k+2-\mu)}(x+1)^{k} .
$$

From the former $N$ interpolation conditions in (5) we know that polynomial

$$
\sum_{k=0}^{N-1} a_{k+1} \frac{(k+1) !}{\Gamma(k+2-\mu)}(x+1)^{k} \in \mathcal{P}_{N-1}
$$

has $N$ zeros. Hence we write $a_{i}=0(1 \leq i \leq N)$. The coefficient $a_{0}$ is zero from the last interpolation condition in (5). Now we conclude that the zero function is uniquely interpolated by the zero polynomial. The solution of the Caputo fractional Birkhoff interpolation problem for $\mu \in(0,1)$ exists uniquely.

In the case $\mu \in(1,2)$, we check

$$
{ }^{C} D^{\mu} p(x)=\sum_{k=0}^{N}{ }^{C} D^{\mu}\left(a_{k}(x+1)^{k}\right)=(x+1)^{2-\mu} \sum_{k=0}^{N-2} a_{k+2} \frac{(k+2) !}{\Gamma(k+3-\mu)}(x+1)^{k} .
$$

and the polynomial

$$
\sum_{k=0}^{N-2} a_{k+2} \frac{(k+2) !}{\Gamma(k+3-\mu)}(x+1)^{k} \in \mathcal{P}_{N-2}
$$

to find $a_{i}=0(2 \leq i \leq N)$ by using the former $N-1$ conditions in (6). The coefficient $a_{0}$ and $a_{1}$ are zero from the last two interpolation conditions in (6). Once more we conclude that the zero function is uniquely interpolated by the zero polynomial. The solution of the Caputo fractional Birkhoff interpolation problem for $\mu \in(1,2)$ exists uniquely.

Similar to the Caputo one, for the Riemann-Liouville fractional derivative operator, we have the following formulas [14]: for $\mu>0$ and not an integer,

$$
D^{\mu}(x+1)^{n}=\frac{n !}{\Gamma(n-\mu+1)}(x+1)^{n-\mu}, \quad n \text { is a nonnegative integer. }
$$

Consider the Riemann-Liouville fractional Birkhoff interpolation. We choose $\left\{(x+1)^{j}\right\}_{j=0}^{N}$ as a basis of vector space $\mathcal{P}_{N}$. Then for $\mu \in(0,1)$, the solvability of the Riemann-Liouville fractional Birkhoff interpolation is equivalent to the invertibility of the following matrix

$$
\left(\begin{array}{ccccc}
1 & 0 & 0 & \cdots & 0 \\
\frac{\left(x_{1}+1\right)^{-\mu}}{\Gamma(1-\mu)} & \frac{\left(x_{1}+1\right)^{1-\mu}}{\Gamma(2-\mu)} & \frac{2\left(x_{1}+1\right)^{2-\mu}}{\Gamma(3-\mu)} & \cdots & \frac{N !\left(x_{1}+1\right)^{N-\mu}}{\Gamma(N-\mu+1)} \\
\frac{\left(x_{2}+1\right)^{-\mu}}{\Gamma(1-\mu)} & \frac{\left(x_{2}+1\right)^{1-\mu}}{\Gamma(2-\mu)} & \frac{2\left(x_{2}+1\right)^{2-\mu}}{\Gamma(3-\mu)} & \cdots & \frac{N !\left(x_{2}+1\right)^{N-\mu}}{\Gamma(N-\mu+1)} \\
\vdots & \vdots & \vdots & \ddots & \vdots \\
\frac{\left(x_{N}+1\right)^{-\mu}}{\Gamma(1-\mu)} & \frac{\left(x_{N}+1\right)^{1-\mu}}{\Gamma(2-\mu)} & \frac{2\left(x_{N}+1\right)^{2-\mu}}{\Gamma(3-\mu)} & \cdots & \frac{N !\left(x_{N}+1\right)^{N-\mu}}{\Gamma(N-\mu+1)}
\end{array}\right) .
$$


We now check the matrix (11). By Laplace's theorem, the determinant of the matrix (11) is equal to determinant of the matrix

$$
\left(\begin{array}{cccc}
\frac{\left(x_{1}+1\right)^{1-\mu}}{\Gamma(2-\mu)} & \frac{2\left(x_{1}+1\right)^{2-\mu}}{\Gamma(3-\mu)} & \cdots & \frac{N !\left(x_{1}+1\right)^{N-\mu}}{\Gamma(N-\mu+1)} \\
\frac{\left(x_{2}+1\right)^{1-\mu}}{\Gamma(2-\mu)} & \frac{2\left(x_{2}+1\right)^{2-\mu}}{\Gamma(3-\mu)} & \cdots & \frac{N !\left(x_{2}+1\right)^{N-\mu}}{\Gamma(N-\mu+1)} \\
\vdots & \vdots & \ddots & \vdots \\
\frac{\left(x_{N}+1\right)^{1-\mu}}{\Gamma(2-\mu)} & \frac{2\left(x_{N}+1\right)^{2-\mu}}{\Gamma(3-\mu)} & \cdots & \frac{N !\left(x_{N}+1\right)^{N-\mu}}{\Gamma(N-\mu+1)}
\end{array}\right)
$$

Cross out the factors $\left(x_{i}+1\right)^{1-\mu}$ at $i-$ th row and $\frac{k !}{\Gamma(k-\mu+1)}$ at $k$-th column in (12), we have a Vandemonde matrix

$$
\left(\begin{array}{cccc}
1 & \left(x_{1}+1\right) & \cdots & \left(x_{1}+1\right)^{N-1} \\
1 & \left(x_{2}+1\right) & \cdots & \left(x_{2}+1\right)^{N-1} \\
\vdots & \vdots & \ddots & \vdots \\
1 & \left(x_{N}+1\right) & \cdots & \left(x_{N}+1\right)^{N-1}
\end{array}\right) .
$$

Hence, making use of the well-known Vandemonde matrix (13), we can derive the determinant of the matrix (11)

$$
\left(\prod_{i=1}^{N}\left(x_{i}+1\right)^{1-\mu}\right)\left(\prod_{i=1}^{N} \frac{i !}{\Gamma(i-\mu+1)}\right)\left(\prod_{1 \leq i<j \leq N}\left(x_{i}-x_{j}\right)\right) .
$$

We claim that the the Riemann-Liouville fractional Birkhoff interpolation problem for $\mu \in(0,1)$ is solvable from the invertibility of the matrix (11) assuming that the interpolating nodes $\left\{x_{i}\right\}_{i=1}^{N}$ are all distinct.

For $\mu \in(1,2)$, we need to check the matrix

$$
\left(\begin{array}{ccccc}
1 & 0 & 0 & \cdots & 0 \\
1 & 2 & 2^{2} & \cdots & 2^{N} \\
\frac{\left(x_{1}+1\right)^{-\mu}}{\Gamma(1-\mu)} & \frac{\left(x_{1}+1\right)^{1-\mu}}{\Gamma(2-\mu)} & \frac{2\left(x_{1}+1\right)^{2-\mu}}{\Gamma(3-\mu)} & \cdots & \frac{N !\left(x_{1}+1\right)^{N-\mu}}{\Gamma(N-\mu+1)} \\
\vdots & \vdots & \vdots & \ddots & \vdots \\
\frac{\left(x_{N-1}+1\right)^{-\mu}}{\Gamma(1-\mu)} & \frac{\left(x_{N-1}+1\right)^{1-\mu}}{\Gamma(2-\mu)} & \frac{2\left(x_{N-1}+1\right)^{2-\mu}}{\Gamma(3-\mu)} & \cdots & \frac{N !\left(x_{N-1}+1\right)^{N-\mu}}{\Gamma(N-\mu+1)}
\end{array}\right) .
$$

It is not true that the matrix (14) is invertible for every $N \geq 1$. For example, when $N=1$, the determinant of the matrix (14) is $\frac{4\left(x_{1}+1\right)^{1-\mu}}{\Gamma(3-\mu)}\left(x_{1}+\mu-1\right)$. This means that when $x_{1} \neq 1-\mu$ the matrix (14) is invertible. Hence, the solvability of the Riemann-Liouville fractional Birkhoff interpolation problem for $\mu \in(1,2)$ is more complicated. The condition of solvability of the Riemann-Liouville fractional Birkhoff interpolation problem for $\mu \in(1,2)$ is unknown.

\section{Approximation Error of Fractional Birkhoff Interpolations}

For error estimate, we focus on Jacobi-Gauss-Lobatto nodes as the interpolating points for all Birkhoff problems concerned in this section. We will analyse the error between the concerned function and its Birkhoff interpolation approximation on Gauss-type interpolating points. Let $J_{m}^{\alpha, \beta}(x)$ be the classical Jacobi orthogonal polynomial of degree $m$ with index pair $(\alpha, \beta)$. We take Jacobi-Gauss-Lobatto nodes as the interpolating points, namely, $\left\{x_{j}=x_{j, N}^{(\alpha, \beta)}\right\}_{j=0}^{N}$, and $x_{0, N}^{(\alpha, \beta)}=-1, x_{N, N}^{(\alpha, \beta)}=1, x_{j, N}^{(\alpha, \beta)}(1 \leq j \leq N-1)$ are the zeros of $\partial_{x} J_{N}^{\alpha, \beta}$ (also zeros of $J_{N-1}^{\alpha+1, \beta+1}$ ).

In order to analyse the concerned interpolation error, we introduce the following Lagrange-Gauss-Radau interpolation $\Pi_{N-1}^{(1)}$, which is defined as: for $u \in C(0,1], \Pi_{N-1}^{(1)} u \in \mathcal{P}_{N-1}$, such that

$$
\Pi_{N-1}^{(1)} u\left(x_{j, N}^{(\alpha, \beta)}\right)=u\left(x_{j, N}^{(\alpha, \beta)}\right), \quad 1 \leq j \leq N .
$$


The following lemma (page 136, Theorem 3.42 in [15]) is important for error estimate.

Lemma 1 For $\alpha>-1, \beta>-2$, and any $u \in B_{\alpha, \beta+1}^{m}(-1,1)$, we have that for $0 \leq l \leq m$ and $\beta+2<m \leq N$,

$$
\left\|\partial_{x}^{l}\left(\Pi_{N-1}^{(1)} u-u\right)\right\|_{\omega^{\alpha+l, \beta+l+1}} \leq c \sqrt{\frac{(N-m) !}{(N-1) !}}(N-1)^{l-(m+1) / 2}\left\|\partial_{x}^{m} u\right\|_{\omega^{\alpha+m, \beta+m+1}},
$$

where $c$ is a positive constant independent of $m, N$ and $u$.

For the Caputo fractional Birkhoff interpolation operator with $\mu \in(0,1)$, we have that

$$
\left({ }^{C} D^{\mu}{ }^{C} I_{N}^{\mu} u\right)(x)=\left(\Pi_{N-1}^{(1)}{ }^{C} D^{\mu} u\right)(x) \in \mathcal{P}_{N-1} .
$$

Now we come to the following error estimation.

Theorem 1 Under the assumption of Lemma 1,

$$
\left\|\partial_{x}^{l}\left[{ }^{C} D^{\mu}\left({ }^{C} I_{N}^{\mu} u-u\right)\right]\right\|_{\omega^{\alpha+l, \beta+l+1}} \leq c \sqrt{\frac{(N-m) !}{(N-1) !}}(N-1)^{l-(m+1) / 2}\left\|\partial_{x}^{m}\left({ }^{C} D^{\mu} u\right)\right\|_{\omega^{\alpha+m, \beta+m+1}},
$$

where $c$ is a positive constant independent of $m, N$ and $u$.

Proof. The result is obtained by combining Lemma 1 and (15).

We need the following Lagrange-Gauss interpolation $\Pi_{N-2}^{(2)}$ which is defined as: for $u \in C(0,1), \Pi_{N-2}^{(2)} u \in$ $\mathcal{P}_{N-2}$, such that

$$
\Pi_{N-2}^{(2)} u\left(x_{j, N}^{(\alpha, \beta)}\right)=u\left(x_{j, N}^{(\alpha, \beta)}\right), \quad 1 \leq j \leq N-1 .
$$

The following lemma (page 133, Theorem 3.41 in [15]) is important for error estimate.

Lemma 2 For $\alpha, \beta>-2$, and any $u \in B_{\alpha+1, \beta+1}^{m}(-1,1)$, we have for $0 \leq l \leq m<N-1$,

$$
\left\|\partial_{x}^{l}\left(\Pi_{N-2}^{(2)} u-u\right)\right\|_{\omega^{\alpha+l+1, \beta+l+1}} \leq c \sqrt{\frac{(N-m-1) !}{(N-2) !}}(N+m-2)^{l-(m+1) / 2}\left\|\partial_{x}^{m} u\right\|_{\omega^{\alpha+m+1, \beta+m+1}},
$$

where $c$ is a positive constant independent of $m, N$ and $u$.

For the Caputo fractional Birkhoff interpolation operator with $\mu \in(1,2)$, we have that

$$
\left({ }^{C} D^{\mu}{ }^{C} I_{N}^{\mu} u\right)(x)=\left(\Pi_{N-2}^{(2)}{ }^{C} D^{\mu} u\right)(x) \in \mathcal{P}_{N-2} .
$$

Now we come to the following error estimation. Similarly, combining the result in Lemma 2 with (16), we obtain

Theorem 2 Under the assumption of Lemma 2,

$$
\begin{aligned}
& \left\|\partial_{x}^{l}\left[{ }^{C} D^{\mu}\left({ }^{C} I_{N}^{\mu} u-u\right)\right]\right\|_{\omega^{\alpha+l+1, \beta+l+1}} \\
\leq & c \sqrt{\frac{(N-m-1) !}{(N-2) !}(N+m-2)^{l-(m+1) / 2}\left\|\partial_{x}^{m}\left({ }^{C} D^{\mu} u\right)\right\|_{\omega^{\alpha+m+1, \beta+m+1}}},
\end{aligned}
$$

where $c$ is a positive constant independent of $m, N$ and $u$.

\section{Concluding Remarks}

Some Birkhoff-type interpolations which are related with first-order initial value problem and second-order boundary value problem of fractional-order differential operators are considered in the paper. As in [12] or [13], this Birkhoff interpolations lead to well-conditioned collocation matrices or pre-conditioner. We remark that it is worthy of consideration on the similar interpolations for higher fractional-order derivative case. For the Riesz fractional-order derivative or other type fractional-order operators, the similar interpolation problems and their theories need further development. 


\section{Acknowledgement}

We thank the Editor and the referee for their comments. The authors were supported by National Natural Science Foundation of China (Grant No. 11661048 and No. 11261027).

\section{References}

1. G. D. Birkhoff, "General mean value and remainder theorems with applications to mechanical differentiation and quadrature," Transactions on American Mathematical Society, vol. 7, no. 1, pp. 107-136, 1906.

2. G. G. Lorentz and K. L. Zeller, "Birkhoff interpolation," SIAM Journal on Numerical Analysis, vol. 8, no. 1, pp. 43-48, 1971.

3. G. G. Lorentz, K. Jetter and S. D. Riemenschneider, Birkhoff Interpolation. Addison-Wesley Publ. Comp., 1983.

4. G. Pólya, "Bemerkung zur interpolation und zur Näherungstheorie der balkenbiegung," Zeitschrift für Angewandte Mathematik und Mechanik, vol. 11, no. 3, pp. 445-449, 1931.

5. I. J. Schoenberg, "On Hermite-Birkhoff interpolation," Journal of Mathematical analysis and applications, vol. 16 , no. 3, pp. 538-543, 1966.

6. A. Sharma, "Some poised and unpoised problems of interpolation," SIAM Review, vol. 14, no. 1, pp. 129-151, 1972.

7. Y. G. Shi, Theory of Birkhoff Interpolation. Nova Science Publ., 2003.

8. D. R. Ferguson, "The question of uniqueness for G. D. Birkhoff interpolation problems," Journal of approximation theory, vol. 2, no. 1, pp.1-28, 1969.

9. F. Palacios and P. Rubió, "Generalised Pólya condition for Birkhoff interpolation with lacunary polynomial," Applied Mathematics E-Notes, vol. 3, no. 1, pp. 124-129, 2003.

10. G. Mühlbach, "An algorithm aproach to Hermite-Birkhoff-interpolation," Numerische Mathematik, vol. 37, no. 3, pp. 339-347, 1981.

11. L. Szili, "A survey on $(0,2)$ interpolation," Annales Universitatis Scientiarum Budapestinensis de Rolando Eötvös Nominatae Sectio Computatorica, vol. 16, no. 1, pp. 377-390, 1996.

12. L. L. Wang, D. D. Samson and X. D. Zhao, "A well-conditioned collocation method using a pseudospectral integration matrix," SIAM Journal on Scientific Computing, vol. 36, no. 3, pp. A907-A929, 2014.

13. Y. J. Jiao, L. L. Wang and C. Huang, "Well-conditioned fractional collocation methods using fractional Birkhoff interpolation basis," Journal of Computational Physics, vol. 305, no. 1, pp. 1-28, 2016.

14. A. A. Kilbas, H. M. Srivastava and J. J. Trujillo, Theory and Applications of Fractional Differential Equations. Elsevier, 2006.

15. J. Shen, T. Tang and L. L. Wang, Spectral methods: Algorithms, Analysis and Applications. Springer Series in Computational Mathematics 41, Springer-Verlag, 2011. 